\title{
A General Setting for the Parametric Google Matrix
}

\author{
Roger A. Horn and Stefano Serra-Capizzano
}

Abstract. The spectral and Jordan structures of the web hyperlink matrix $G(c)=$ $c G+(1-c) e v^{T}$ have been analyzed when $G$ is the basic (stochastic) Google matrix, $c$ is a real parameter such that $0<c<1, v$ is a nonnegative probability vector, and $e$ is the all-ones vector. Typical studies have relied heavily on special properties of nonnegative, positive, and stochastic matrices. There is a unique nonnegative vector $y(c)$ such that $y(c)^{T} G(c)=y(c)^{T}$ and $y(c)^{T} e=1$. This PageRank vector $y(c)$ can be computed effectively by the power method.

We consider a square complex matrix $A$ and nonzero complex vectors $x$ and $v$ such that $A x=\lambda x$ and $v^{*} x=1$. We use standard matrix analytic tools to determine the eigenvalues, the Jordan blocks, and a distinguished left $\lambda$-eigenvector of $A(c)=$ $c A+(1-c) \lambda x v^{*}$ as a function of a complex variable $c$. If $\lambda$ is a semisimple eigenvalue of $A$, there is a uniquely determined projection $P$ such that $\lim _{c \rightarrow 1} y(c)=P v$ for every $v$ such that $v^{*} x=1$; this limit may fail to exist for some $v$ if $\lambda$ is not semisimple. As a special case of our results, we obtain a complex analog of PageRank for the web hyperlink matrix $G(c)$ with a complex parameter $c$. We study regularity, limits, expansions, and conditioning of $y(c)$, and we propose a complex extrapolation algorithm that may provide an efficient way to compute PageRank.

\section{Introduction}

The web can be regarded as a huge directed graph whose $n$ nodes are webpages and whose edges are the links between pages [Langville and Meyer 04]. Let $\operatorname{deg}(i)$ denote the number of pages different from $i$ that can be reached by a direct link from page $i$. The basic Google matrix $G=\left[G_{i j}\right]$ is defined as follows: If $\operatorname{deg}(i)>0$, then $G_{i j}=1 / \operatorname{deg}(i)$ for each page $j \neq i$ that can be reached by a direct link from page $i$; all other entries of $G$ in row $i$ are zero. If $\operatorname{deg}(i)=0$

(C) A K Peters, Ltd.

I542-795I/06 \$0.50 per page 
(a dangling node), then $G_{i j}=1 / n$ for all $j=1, \ldots, n$. This definition corresponds to the following web user behavior: a user visiting page $i$ moves, if $\operatorname{deg}(i)>0$, with probability $1 / \operatorname{deg}(i)$ to one of the $\operatorname{deg}(i)$ pages linked to page $i$ and, if $\operatorname{deg}(i)=0$, with probability $1 / n$ to any page in the web.

Since all of the row sums of $G$ are 1 , the $n$-vector $e$ of all ones is a right eigenvector of $G$ associated with the eigenvalue 1, that is, $G e=e$. Since all the entries of $G$ are nonnegative, the Perron-Frobenius Theorem ensures that no eigenvalue of $G$ has modulus greater than 1 and that there is a nonnegative $n$-vector $y$ such that $y^{T} G=y^{T}$ and $y^{T} e=1 ; y^{T}$ denotes the transpose of $y$.

PageRank is a nonnegative $n$-vector whose individual entries are interpreted as a measure of importance of the webpages corresponding to them. One way to define the importance of a given page is to let it be the limit probability that a generic user reaches that page after infinitely many clicks. In this surfing model of the web, PageRank is a nonnegative left eigenvector $y$ of $G$ associated with the eigenvalue 1 , that is, $y^{T} G=y^{T}$ (normalized so that $y^{T} e=1$ ).

Ideally, to compute PageRank one would like to compute a normalized nonnegative solution of the system of linear equations $y^{T} G=y^{T}$. The good news is that a solution always exists; the bad news is that there might be multiple independent solutions. And even if there is a unique solution, computing it by standard methods such as the power method may fail because $G$ has one or more eigenvalues different from 1 that have modulus 1 [Golub and Van Loan 83, Langville and Meyer 05]. The standard resolution of these difficulties is to modify $G$ : for a given $c \in[0,1]$ and a given nonnegative $n$-vector $v$ such that $v^{T} e=1$, define the parametric Google matrix

$$
G(c)=c G+(1-c) e v^{T},
$$

which corresponds to the following user behavior: with probability $c$, a user visiting page $i$ moves according to the rule described by the basic Google matrix $G$; with probability $1-c$ the user moves according to the rule described by the nonnegative probability vector $v=\left[v_{j}\right]$ (that is, moves to page $j$ with probability $v_{j}$ ). The value $c=0.85$ is often considered in the literature. (See [Langville and Meyer 04] and the references therein.) If $0 \leq c<1$, it turns out that 1 is the only eigenvalue of $G(c)$ that has modulus 1 and it is associated with a unique nonnegative normalized left eigenvector $y(c)$, which can be computed reliably by the power method; see [Langville and Meyer 04] and the references therein. Indeed, the error in the computed value of $y(c)$ at step $k$ is at most a fixed constant times $c^{k}$. Of course, the computation could be quite slow if $c$ is very close to 1 .

We have three expository goals. The first is to describe the eigenvalues and Jordan blocks of $G(c)$ as a function of a complex parameter $c$; we begin by analyzing a class of complex matrices that contains $G(c)$ as a special case. Second, 
we want to explain how one can analyze the behavior (regularity, limits, expansions, conditioning, etc.) of the normalized left eigenvector $y(c)$ of $G(c)$ as a function of the complex variable $c$. Finally, we describe an algorithm to compute PageRank that exploits the complex analytical properties of $y(c)$.

The paper is organized as follows. In Section 2, we set notation and terminology for the basic matrix-theoretic concepts that we employ to analyze a generalization of $G(c)$ : for a square complex matrix $A$, nonzero complex vectors $x$ and $v$ such that $A x=\lambda x$ and $v^{*} x=1$, and a complex variable $c$, we study $A(c)=c A+(1-c) \lambda x v^{*}$. In Section 3 we explain how Alfred Brauer used the classical principle of biorthogonality in 1952 to prove a theorem that reveals the eigenvalues of $A(c)$. In Section 4 we introduce the complete principle of biorthogonality and use it to obtain the Jordan blocks of $A(c)$ under the assumption that there is a nonzero vector $y$ such that $y^{*} A=\lambda y^{*}$ and $y^{*} x=1$. In particular, such a vector $y$ exists if $\lambda$ is a simple or semisimple eigenvalue of $A$. In Section 5 we derive a representation for a distinguished left $\lambda$-eigenvector $y(c)$ of $A(c)$; this representation is an explicit rational vector-valued function of the complex variable $c$. In Sections 6 and 7 we study $\lim _{c \rightarrow 1} y(c)$ and the associated projection, respectively, and in Section 8 we focus on the special case in which $A$ is the basic Google matrix $G, x=e$, and $v$ is a nonnegative probability vector. In Section 9, we propose an algorithm to compute PageRank that exploits properties of $G(c)$ as a function of a complex variable $c$. Our final section mentions some prior work.

\section{Terminology and Notation}

All the matrices and vectors that we consider have real or complex entries. We denote the conjugate transpose of an $m \times n$ matrix $X=\left[x_{i j}\right]$ by $X^{*}=\left[\bar{x}_{j i}\right]$. If $A$ is a square matrix, its characteristic polynomial is $p_{A}(t):=\operatorname{det}(t I-A)$; the (complex) zeroes of $p_{A}(t)$ are the eigenvalues of $A$. A complex number $\lambda$ is an eigenvalue of $A$ if and only if there are nonzero vectors $x$ and $y$ such that $A x=\lambda x$ and $y^{*} A=\lambda y^{*} ; x$ is said to be an eigenvector (more specifically, a right eigenvector) of $A$ associated with $\lambda$ and $y$ is said to be a left eigenvector of $A$ associated with $\lambda$. If $\lambda$ is an eigenvalue of $A$, its algebraic multiplicity is its multiplicity as a zero of $p_{A}(t)$; its geometric multiplicity is the maximum number of linearly independent eigenvectors associated with it. The geometric multiplicity of an eigenvalue is never greater than its algebraic multiplicity. An eigenvalue whose algebraic multiplicity is one is said to be simple. An eigenvalue whose algebraic and geometric multiplicities are equal is said to be semisimple; an eigenvalue $\lambda$ of $A$ is semisimple if and only if $\operatorname{rank}(A-\lambda I)=\operatorname{rank}(A-\lambda I)^{2}$.

We let $e_{1}$ indicate the first column of the identity matrix $I: e_{1}=\left[\begin{array}{llll}1 & 0 & \ldots & 0\end{array}\right]^{T}$. We let $e=\left[\begin{array}{llll}1 & 1 & \ldots & 1\end{array}\right]^{T}$ denote the all-ones vector. Whenever it is useful to indicate that an identity or zero matrix has a specific size, e.g., $r \times r$, we write $I_{r}$ or $0_{r}$. 
Two vectors $x$ and $y$ of the same size are orthogonal if $x^{*} y=0$. The orthogonal complement of a given set of vectors is the set (actually, a vector space) of all vectors that are orthogonal to every vector in the given set.

An $n \times r$ matrix $X$ has orthonormal columns if $X^{*} X=I_{r}$. A square matrix $U$ is unitary if it has orthonormal columns, that is, if $U^{*}$ is the inverse of $U$.

A square matrix $A$ is a projection if $A^{2}=A$. A square matrix $A$ is row stochastic if it has real nonnegative entries and $A e=e$, which means that the sum of the entries in each row is $1 ; A$ is column stochastic if $A^{T}$ is row stochastic. We say that $A$ is stochastic if it is either row stochastic or column stochastic.

The direct sum of $k$ given square matrices $X_{1}, \ldots, X_{k}$ is the block diagonal matrix

$$
\left[\begin{array}{ccc}
X_{1} & \cdots & 0 \\
\vdots & \ddots & \vdots \\
0 & \cdots & X_{k}
\end{array}\right]=X_{1} \oplus \cdots \oplus X_{k} .
$$

The $k \times k$ Jordan block with eigenvalue $\lambda$ is

$$
J_{k}(\lambda)=\left[\begin{array}{cccc}
\lambda & 1 & & 0 \\
& \ddots & \ddots & \\
& & \ddots & 1 \\
0 & & & \lambda
\end{array}\right], \quad J_{1}(\lambda)=[\lambda]
$$

Each square complex matrix $A$ is similar to a direct sum of Jordan blocks, which is unique up to permutation of the blocks; this direct sum is the Jordan canonical form of $A$. The algebraic multiplicity of $\lambda$ as an eigenvalue of $J_{k}(\lambda)$ is $k$; its geometric multiplicity is 1 . If $\lambda$ is a semisimple eigenvalue of $A$ with multiplicity $m$, then the Jordan canonical form of $A$ is $\lambda I_{m} \oplus J$, in which $J$ is a direct sum of Jordan blocks with eigenvalues different from $\lambda$; if $\lambda$ is a simple eigenvalue, then $m=1$ and the Jordan canonical form of $A$ is $[\lambda] \oplus J$.

The spectral radius of $A$ is $\rho(A)=\max \{|\lambda|: \lambda$ is an eigenvalue of $A\}$. For any complex $n$-vector $x=\left[x_{i}\right]$, its $\ell_{1}$ norm is $\|x\|_{1}=\left|x_{1}\right|+\cdots+\left|x_{n}\right|$.

Suppose that a square matrix $A$ is similar to the direct sum of a zero matrix and a nonsingular matrix, that is,

$$
A=S\left[\begin{array}{cc}
0_{m} & 0 \\
0 & B
\end{array}\right] S^{-1}, B \text { is nonsingular. }
$$

The matrix

$$
A^{D}=S\left[\begin{array}{cc}
0_{m} & 0 \\
0 & B^{-1}
\end{array}\right] S^{-1}
$$

is called the Drazin inverse of $A$; it does not depend on the choice of $S$ or $B$ in the representation (2.1) [Campbell and Meyer 79, Chapter 7]. Moreover, 
both $A A^{D}=A^{D} A$ and $I-A A^{D}$ are projections. If $X$ and $Y$ have $m$ columns, $S=\left[X S_{2}\right]$, and $\left(S^{-1}\right)^{*}=\left[\begin{array}{ll}Y & Z_{2}\end{array}\right]$, then $A^{D}=S_{2} B^{-1} Z_{2}^{*}$ and $I-A A^{D}=X Y^{*}$.

In a block matrix, the symbol $\star$ denotes a block whose entries are not required to take particular values.

For a systematic discussion of the terms defined in this section as well as a broad range of matrix analysis issues, see [Horn and Johnson 85].

\section{Basic Biorthogonality and Eigenvalues}

The following observation about left and right eigenvectors associated with different eigenvalues is the basic principle of biorthogonality [Horn and Johnson 85, Theorem 1.4.7].

Lemma 3.I. Let $A$ be a square complex matrix, and let $x$ and $y$ be nonzero complex vectors such that $A x=\lambda x$ and $y^{*} A=\mu y^{*}$. If $\lambda \neq \mu$, then $y^{*} x=0$ (that is, $x$ and $y$ are orthogonal).

Proof. Compute $y^{*} A x$ in two ways: (i) as $y^{*}(A x)=y^{*}(\lambda x)=\lambda\left(y^{*} x\right)$, and (ii) as $\left(y^{*} A\right) x=\left(\mu y^{*}\right) x=\mu\left(y^{*} x\right)$. Since $\lambda\left(y^{*} x\right)=\mu\left(y^{*} x\right)$ and $\lambda \neq \mu$, it follows that $y^{*} x=0$.

For a given vector $v$ and a matrix $A$ with eigenvalue $\lambda$ and associated eigen-

vector $x$, how are the eigenvalues of $A+x v^{*}$ related to those of $A$ ? This question was asked and answered by Alfred Brauer in 1952 [Brauer 52, Theorem 26]:

Theorem 3.2. (Brauer.) Let $A$ be an $n \times n$ complex matrix, and let $x$ be a nonzero complex vector such that $A x=\lambda x$. Let

$$
\lambda, \lambda_{2}, \ldots, \lambda_{n}
$$

be the eigenvalues of $A$. Then, for any complex $n$-vector $v$, the eigenvalues of $A+x v^{*}$ are

$$
\lambda+v^{*} x, \lambda_{2}, \ldots, \lambda_{n}
$$

Brauer's proof involved three steps:

1. Compute

$$
\left(A+x v^{*}\right) x=A x+x v^{*} x=\lambda x+\left(v^{*} x\right) x=\left(\lambda+v^{*} x\right) x,
$$

which shows that $\lambda+v^{*} x$ is an eigenvalue of $A+x v^{*}$. 
2. If $\mu$ is an eigenvalue of $A$ that is different from $\lambda$, and if $y$ is a left eigenvector of $A$ associated with $\mu$, then Lemma 3.1 ensures that

$$
y^{*}\left(A+x v^{*}\right)=y^{*} A+y^{*} x v^{*}=\mu y+\left(y^{*} x\right) v=\mu y+0 \cdot v=\mu y .
$$

Thus, the distinct eigenvalues of $A$ that are different from $\lambda$ are all eigenvalues of $A+x v^{*}$, but perhaps not with the same multiplicities.

3. Use a continuity argument to show that the multiplicities of the common eigenvalues of $A$ and $A+x v^{*}$ (setting aside the respective eigenvalues $\lambda$ and $\left.\lambda+v^{*} x\right)$ are the same.

Brauer's theorem tells us something interesting about the eigenvalues of $A(c)$.

Corollary 3.3. Let $A$ be an $n \times n$ complex matrix. Let $\lambda$ be an eigenvalue of $A$, let $x$ and $v$ be nonzero complex vectors such that $A x=\lambda x$ and $v^{*} x=1$, and let $A(c)=c A+(1-c) \lambda x v^{*}$. Let $\lambda, \lambda_{2}, \ldots, \lambda_{n}$ be the eigenvalues of $A$. Then, for any complex number $c$, the eigenvalues of $A(c)$ are $\lambda, c \lambda_{2}, \ldots, c \lambda_{n}$.

Proof. In the statement of Brauer's Theorem, replace $A$ and $v$ by $c A$ and $(1-\bar{c}) \bar{\lambda} v$, respectively. The eigenvalues of $c A$ are $c \lambda, c \lambda_{2}, \ldots, c \lambda_{n}, x$ is an eigenvector of $c A$ associated with the eigenvalue $c \lambda$, and Brauer's Theorem tells us that the eigenvalues of $c A+x((1-\bar{c}) \bar{\lambda} v)^{*}=c A+(1-c) \lambda x v^{*}$ are $c \lambda+(1-c) \lambda v^{*} x, c \lambda_{2}, \ldots, c \lambda_{n}$, which are $\lambda, c \lambda_{2}, \ldots, c \lambda_{n}$ since $v^{*} x=1$.

Robert Reams revisited Brauer's theorem in 1996 [Reams 96, p. 368]. He observed that the Schur triangularization theorem [Horn and Johnson 85, Theorem 2.3.1] can be used to prove Brauer's Theorem without a continuity argument: Let $S=\left[x S_{1}\right]$ be any nonsingular matrix that upper triangularizes $A$ as

$$
S^{-1} A S=\left[\begin{array}{cccc}
\lambda & \star & \cdots & \star \\
& \lambda_{2} & \ddots & \vdots \\
& & \ddots & \star \\
0 & & & \lambda_{n}
\end{array}\right]
$$

and whose first column is an eigenvector $x$ associated with the eigenvalue $\lambda$. Since $I=S^{-1} S=\left[\begin{array}{ll}S^{-1} x & \star\end{array}\right]$, we see that $S^{-1} x=e_{1}$. Compute

$$
\begin{aligned}
S^{-1}\left(x v^{*}\right) S & =\left(S^{-1} x\right)\left(v^{*} S\right)=\left[\begin{array}{c}
1 \\
0 \\
\vdots \\
0
\end{array}\right]\left[\begin{array}{llll}
v^{*} x & \star & \cdots & \star
\end{array}\right] \\
& =\left[\begin{array}{cccc}
v^{*} x & \star & \cdots & \star \\
0 & 0 & \cdots & 0 \\
\vdots & \vdots & \ddots & 0 \\
0 & 0 & \cdots & 0
\end{array}\right]
\end{aligned}
$$


Therefore, the similarity

$$
S^{-1}\left(A+x v^{*}\right) S=\left[\begin{array}{cccc}
\lambda+v^{*} x & \star & \cdots & \star \\
0 & \lambda_{2} & \ddots & \vdots \\
\vdots & \ddots & \ddots & \star \\
0 & \cdots & 0 & \lambda_{n}
\end{array}\right]
$$

reveals both the eigenvalues of $A+x v^{*}$ and their multiplicities.

\section{Complete Biorthogonality and Jordan Blocks}

Brauer used the basic principle of biorthogonality to analyze the eigenvalues of $A+x v^{*}$. We now want to analyze the Jordan blocks of $A+x v^{*}$.

The basic principle of biorthogonality is silent about what happens when $\lambda=$ $\mu$. In that event, there are three possibilities:

1. $y^{*} x=0$ (we can normalize so that $x^{*} x=y^{*} y=1$ );

2. $y^{*} x \neq 0$ (we can normalize so that $y^{*} x=1$ ); or

3. $x=\alpha y$ (we can normalize so that $x=y$ and $x^{*} x=1$ ).

The following complete principle of biorthogonality addresses all the possibilities and describes reduced forms for $A$ that can be achieved in each case.

Theorem 4.I. Let $A$ be an $n \times n$ complex matrix, and let $x$ and $y$ be nonzero complex vectors such that $A x=\lambda x$ and $y^{*} A=\mu y^{*}$.

(a) Suppose that $\lambda \neq \mu$ and $x^{*} x=y^{*} y=1$. Then, $y^{*} x=0$. Let $U=$ $\left[\begin{array}{lll}x & y & U_{1}\end{array}\right]$, in which the columns of $U_{1}$ are any given orthonormal basis for the orthogonal complement of $x$ and $y$. Then, $U$ is unitary and

$$
U^{*} A U=\left[\begin{array}{ccc}
\lambda & \star & \star \\
0 & \mu & 0 \\
0 & \star & B
\end{array}\right], \quad B=U_{1}^{*} A U_{1} \text { is }(n-2) \times(n-2) .
$$

(b) Suppose that $\lambda=\mu, y^{*} x=0$, and $x^{*} x=y^{*} y=1$. Let $U=\left[\begin{array}{lll}x & y & U_{1}\end{array}\right]$, in which the columns of $U_{1}$ are any given orthonormal basis for the orthogonal complement of $x$ and $y$. Then, $U$ is unitary, the algebraic multiplicity of $\lambda$ is at least two, and

$$
U^{*} A U=\left[\begin{array}{ccc}
\lambda & \star & \star \\
0 & \lambda & 0 \\
0 & \star & B
\end{array}\right], \quad B=U_{1}^{*} A U_{1} \text { is }(n-2) \times(n-2) .
$$


(c) Suppose that $\lambda=\mu$ and $y^{*} x=1$. Let $S=\left[x S_{1}\right]$, in which the columns of $S_{1}$ are any given basis for the orthogonal complement of $y$. Then, $S$ is nonsingular, $\left(S^{-1}\right)^{*}=\left[y Z_{1}\right]$, the columns of $Z_{1}$ are a basis for the orthogonal complement of $x$, and

$$
S^{-1} A S=\left[\begin{array}{cc}
\lambda & 0 \\
0 & B
\end{array}\right], \quad B=Z_{1}^{*} A S_{1} \text { is }(n-1) \times(n-1) .
$$

(d) Suppose that $\lambda=\mu, x=y$, and $x^{*} x=1$. Let $U=\left[x U_{1}\right]$, in which the columns of $U_{1}$ are any given orthonormal basis for the orthogonal complement of $x$. Then, $U$ is unitary and

$$
U^{*} A U=\left[\begin{array}{cc}
\lambda & 0 \\
0 & B
\end{array}\right], \quad B=U_{1}^{*} A U_{1} \text { is }(n-1) \times(n-1) .
$$

\section{Proof.}

(a) Lemma 3.1 ensures that $x$ and $y$ are orthogonal. Let $U=\left[\begin{array}{lll}x & y & U_{1}\end{array}\right]$, in which the columns of $U_{1}$ are a given orthonormal basis for the orthogonal complement of $x$ and $y$. The $n$ columns of $U$ are an orthonormal set, so $U$ is unitary. Compute the unitary similarity

$$
\begin{aligned}
U^{*} A U & \left.=\left[\begin{array}{c}
x^{*} \\
y^{*} \\
U_{1}^{*}
\end{array}\right] \text { A } \begin{array}{lll}
x & y & U_{1}
\end{array}\right]=\left[\begin{array}{ccc}
x^{*} A x & x^{*} A y & x^{*} A U_{1} \\
y^{*} A x & y^{*} A y & y^{*} A U_{1} \\
U_{1}^{*} A x & U_{1}^{*} A y & U_{1}^{*} A U_{1}
\end{array}\right] \\
& =\left[\begin{array}{ccc}
\lambda x^{*} x & x^{*} A y & x^{*} A U_{1} \\
\lambda y^{*} x & \mu y^{*} y & \mu y^{*} U_{1} \\
\lambda U_{1}^{*} x & U_{1}^{*} A y & U_{1}^{*} A U_{1}
\end{array}\right]=\left[\begin{array}{ccc}
\lambda & \star & \star \\
0 & \mu & 0 \\
0 & \star & U_{1}^{*} A U_{1}
\end{array}\right] .
\end{aligned}
$$

(b) As in (a), construct a unitary matrix $U=\left[\begin{array}{lll}x & y & U_{1}\end{array}\right]$, in which the columns of $U_{1}$ are a given orthonormal basis for the orthogonal complement of $x$ and $y$. The reduced form of $A$ under unitary similarity via $U$ is the same as in (a), but with $\lambda=\mu$. The characteristic polynomial of $A$ is

$$
p_{A}(t)=\operatorname{det}(t I-A)=\operatorname{det}\left[\begin{array}{ccc}
t-\lambda & \star & \star \\
0 & t-\lambda & 0 \\
0 & \star & t I-B
\end{array}\right] .
$$

A Laplace expansion by minors down the first column gives

$$
p_{A}(t)=(t-\lambda) \operatorname{det}\left[\begin{array}{cc}
t-\lambda & 0 \\
\star & t I-B
\end{array}\right]
$$

Finally, a Laplace expansion by minors across the first row gives

$$
p_{A}(t)=(t-\lambda)^{2} \operatorname{det}(t I-B)=(t-\lambda)^{2} p_{B}(t),
$$

so $\lambda$ is a zero of $p_{A}(t)$ with multiplicity at least two. 
(c) Let the columns of $S_{1}$ be a given basis for the orthogonal complement of $y$, and let $S=\left[x S_{1}\right]$. The columns of $S_{1}$ are linearly independent, so $S$ is singular only if $x$ is a linear combination of the columns of $S_{1}$, that is, only if $x=S_{1} \xi$ for some vector $\xi$. But then, $1=y^{*} x=y^{*} S_{1} \xi=0 \xi=0$. This contradiction shows that $S$ is nonsingular. Partition $\left(S^{-1}\right)^{*}=\left[\eta Z_{1}\right]$ and compute

$$
I=S^{-1} S=\left[\begin{array}{c}
\eta^{*} \\
Z_{1}^{*}
\end{array}\right]\left[\begin{array}{ll}
x & S_{1}
\end{array}\right]=\left[\begin{array}{cc}
\eta^{*} x & \eta^{*} S_{1} \\
Z_{1}^{*} x & Z_{1}^{*} S_{1}
\end{array}\right]=\left[\begin{array}{cc}
1 & 0 \\
0 & I_{n-1}
\end{array}\right]
$$

Thus, the $n-1$ columns of $Z_{1}$, necessarily linearly independent, are orthogonal to $x$, so they are a basis for the orthogonal complement of $x$. Also, $\eta^{*} S_{1}=0$ means that $\eta$ is orthogonal to the orthogonal complement of $y$, so $\eta=\alpha y$. But, $1=\eta^{*} x=(\alpha y)^{*} x=\bar{\alpha} y^{*} x=\bar{\alpha}$, so $\alpha=1$ and $\eta=y$. Finally, compute the similarity

$$
\begin{aligned}
S^{-1} A S & =\left[\begin{array}{c}
y^{*} \\
Z_{1}^{*}
\end{array}\right] A\left[\begin{array}{ll}
x & S_{1}
\end{array}\right]=\left[\begin{array}{cc}
y^{*} A x & y^{*} A S_{1} \\
Z_{1}^{*} A x & Z_{1}^{*} A S_{1}
\end{array}\right] \\
& =\left[\begin{array}{cc}
\lambda y^{*} x & \lambda y^{*} S_{1} \\
\lambda Z_{1}^{*} x & Z_{1}^{*} A S_{1}
\end{array}\right]=\left[\begin{array}{cc}
\lambda & 0 \\
0 & Z_{1}^{*} A S_{1}
\end{array}\right] .
\end{aligned}
$$

(d) Let the columns of $U_{1}$ be a given orthonormal basis for the orthogonal complement of $x$. Then, the $n$ columns of $U=\left[\begin{array}{ll}x & U_{1}\end{array}\right]$ are an orthonormal set, so $U$ is unitary. Compute the unitary similarity

$$
\begin{aligned}
U^{*} A U & =\left[\begin{array}{c}
x^{*} \\
U_{1}^{*}
\end{array}\right] A\left[\begin{array}{cc}
x & U_{1}
\end{array}\right]=\left[\begin{array}{cc}
x^{*} A x & x^{*} A U_{1} \\
U_{1}^{*} A x & U_{1}^{*} A U_{1}
\end{array}\right] \\
& =\left[\begin{array}{cc}
\lambda x^{*} x & \lambda x^{*} U_{1} \\
\lambda U_{1}^{*} x & U_{1}^{*} A U_{1}
\end{array}\right]=\left[\begin{array}{cc}
\lambda & 0 \\
0 & U_{1}^{*} A U_{1}
\end{array}\right] .
\end{aligned}
$$

We now use the complete principle of biorthogonality to establish an analog of Theorem 3.2 for Jordan blocks.

Theorem 4.2. Let $A$ be an $n \times n$ complex matrix. Let $\lambda, \lambda_{2}, \ldots, \lambda_{n}$ be the eigenvalues of $A$, and let $x$ and $y$ be nonzero complex vectors such that $A x=\lambda x$ and $y^{*} A=$ $\lambda y^{*}$. Assume that $y^{*} x=1$. Then, the Jordan canonical form of $A$ is

$$
[\lambda] \oplus J_{n_{1}}\left(\nu_{1}\right) \oplus \cdots \oplus J_{n_{k}}\left(\nu_{k}\right)
$$

for some positive integers $k, n_{1}, \ldots, n_{k}$ and some set of eigenvalues $\left\{\nu_{1}, \ldots, \nu_{k}\right\} \subset$ $\left\{\lambda_{2}, \ldots, \lambda_{n}\right\}$. For any complex vector $v$ such that $\lambda+v^{*} x \neq \lambda_{j}$ for each $j=$ $2, \ldots, n$, the Jordan canonical form of $A+x v^{*}$ is

$$
\left[\lambda+v^{*} x\right] \oplus J_{n_{1}}\left(\nu_{1}\right) \oplus \cdots \oplus J_{n_{k}}\left(\nu_{k}\right)
$$


Proof. The hypotheses and Theorem 4.1(c) ensure that

$$
S^{-1} A S=\left[\begin{array}{cc}
\lambda & 0 \\
0 & B
\end{array}\right]
$$

for some nonsingular $S$ of the form $S=\left[x S_{1}\right]$, so that $S^{-1} x=e_{1}$. The eigenvalues of $B$ are $\lambda_{2}, \ldots, \lambda_{n}$; let

$$
J_{n_{1}}\left(\nu_{1}\right) \oplus \cdots \oplus J_{n_{k}}\left(\nu_{k}\right)
$$

be the Jordan canonical form of $B$. Just as in Reams's proof of Brauer's Theorem, we have

$$
S^{-1}\left(x v^{*}\right) S=\left(S^{-1} x\right)\left(v^{*} S\right)=e_{1}\left[\begin{array}{cc}
v^{*} x & v^{*} S_{1}
\end{array}\right]=\left[\begin{array}{cc}
v^{*} x & w^{*} \\
0 & 0
\end{array}\right],
$$

in which we set $w^{*}:=v^{*} S_{1}$. Combining the similarities (4.5) and (4.6), we see that

$$
S^{-1}\left(A+x v^{*}\right) S=\left[\begin{array}{cc}
\lambda+v^{*} x & w^{*} \\
0 & B
\end{array}\right] .
$$

Now let $\xi$ be any given $(n-1)$-vector, verify that

$$
\left[\begin{array}{cc}
1 & \xi^{*} \\
0 & I
\end{array}\right]^{-1}=\left[\begin{array}{cc}
1 & -\xi^{*} \\
0 & I
\end{array}\right],
$$

and compute the similarity

$$
\begin{aligned}
{\left[\begin{array}{cc}
1 & -\xi^{*} \\
0 & I
\end{array}\right]\left[\begin{array}{cc}
\lambda+v^{*} x & w^{*} \\
0 & B
\end{array}\right]\left[\begin{array}{cc}
1 & \xi^{*} \\
0 & I
\end{array}\right]=} & \\
& {\left[\begin{array}{cc}
\lambda+v^{*} x & w^{*}+\xi^{*}\left(\left(\lambda+v^{*} x\right) I-B\right) \\
0 & B
\end{array}\right] }
\end{aligned}
$$

We have assumed that $\lambda+v^{*} x$ is not an eigenvalue of $B$, so we may take

$$
\xi^{*}:=-w^{*}\left(\left(\lambda+v^{*} x\right) I-B\right)^{-1},
$$

in which case $w^{*}+\xi^{*}\left(\left(\lambda+v^{*} x\right) I-B\right)=0$ and $A+x v^{*}$ is revealed to be similar to

$$
\left[\begin{array}{cc}
\lambda+v^{*} x & 0 \\
0 & B
\end{array}\right] .
$$

Thus, the Jordan canonical form of $A+x v^{*}$ is (4.4): the direct sum of $\left[\lambda+v^{*} x\right]$ and the Jordan canonical form of $B$. 
The following result strengthens the conclusion of Corollary 3.3 to describe not only the eigenvalues of $A(c)$ but also its Jordan blocks.

Corollary 4.3. Let $A$ be an $n \times n$ complex matrix. Let $\lambda, \lambda_{2}, \ldots, \lambda_{n}$ be the eigenvalues of $A$; let $x, y$, and $v$ be nonzero complex vectors such that $A x=\lambda x, y^{*} A=\lambda y^{*}$, and $v^{*} x=1$; and let $A(c)=c A+(1-c) \lambda x v^{*}$. Assume that $y^{*} x=1$. Let the Jordan canonical form of $A$ be

$$
[\lambda] \oplus J_{n_{1}}\left(\nu_{1}\right) \oplus \cdots \oplus J_{n_{k}}\left(\nu_{k}\right) .
$$

Then, for any nonzero complex number $c$ such that

$$
c \lambda_{j} \neq \lambda \text { for each } j=2, \ldots, n,
$$

the Jordan canonical form of $A(c)$ is

$$
[\lambda] \oplus J_{n_{1}}\left(c \nu_{1}\right) \oplus \cdots \oplus J_{n_{k}}\left(c \nu_{k}\right) .
$$

Proof. We proceed as in the proof of Corollary 3.3. In the statement of Theorem 4.2 , replace $A$ and $v$ by $c A$ and $(1-\bar{c}) \bar{\lambda} v$, respectively. For any $c, c A$ is similar to

$$
[c \lambda] \oplus c J_{n_{1}}\left(\nu_{1}\right) \oplus \cdots \oplus c J_{n_{k}}\left(\nu_{k}\right),
$$

but if $c \neq 0$, we can say more: this direct sum is similar to

$$
[c \lambda] \oplus J_{n_{1}}\left(c \nu_{1}\right) \oplus \cdots \oplus J_{n_{k}}\left(c \nu_{k}\right) .
$$

Moreover, $x$ is an eigenvector of $c A$ associated with the eigenvalue $c \lambda$, the remaining eigenvalues of $c A$ are $c \lambda_{2}, \ldots, c \lambda_{n}$, and

$$
c \lambda+((1-\bar{c}) \bar{\lambda} v)^{*} x=c \lambda+(1-c) \lambda v^{*} x=c \lambda+(1-c) \lambda=\lambda .
$$

Thus, our assumption (4.7) and Theorem 4.2 ensure that the Jordan canonical form of

$$
c A+x((1-\bar{c}) \bar{\lambda} v)^{*}=c A+(1-c) \lambda x v^{*}=A(c)
$$

is

$$
[\lambda] \oplus J_{n_{1}}\left(c \nu_{1}\right) \oplus \cdots \oplus J_{n_{k}}\left(c \nu_{k}\right)
$$

\section{The Normalized Left $\lambda$-Eigenvector of $A(c)$}

If $\lambda \neq 0$, Corollary 3.3 ensures that it is a simple eigenvalue of $A(c)$ for all but finitely many values of $c$. We would like to have an explicit expression for its associated left eigenvector $y(c)$, normalized so that $y(c)^{*} x=1$. 
Theorem 5.I. Let $A$ be an $n \times n$ complex matrix. Let $\lambda, \lambda_{2}, \ldots, \lambda_{n}$ be the eigenvalues of $A$; let $\mu_{1}, \ldots, \mu_{d}$ be the nonzero eigenvalues of $A$ that are different from $\lambda$; let $x$ and $v$ be nonzero complex vectors such that $A x=\lambda x$ and $v^{*} x=1$; and let $A(c)=c A+(1-c) \lambda x v^{*}$. Assume that $\lambda \neq 0$.

(a) Suppose that there is a complex vector $y$ such that $y^{*} A=\lambda y^{*}$ and $y^{*} x=1$. Assume that $c \lambda_{j} \neq \lambda$ for each $j=2, \ldots, n$. Let $S_{1}, Z_{1}$, and $B$ be defined as in Theorem 4.1(c). Then, $\lambda$ is not an eigenvalue of $c B$. Define the vector $y(c)$ by

$$
y(c)^{*}=y^{*}+(1-c) \lambda v^{*} S_{1}\left(\lambda I_{n-1}-c B\right)^{-1} Z_{1}^{*} .
$$

Then, $y(c)$ satisfies the conditions

$$
y(c)^{*} A(c)=\lambda y(c)^{*} \text { and } y(c)^{*} x=1
$$

If, in addition, $c \neq 1$, then $y(c)$ is the only vector that satisfies the conditions (5.2). If $\lambda$ is a simple eigenvalue of $A$, then it is not an eigenvalue of $B$.

(b) Suppose that $\lambda$ is a semisimple eigenvalue of $A$ with multiplicity $m \geq 2$, and suppose that

$$
c \mu_{j} \neq \lambda \text { for each } j=1, \ldots, d \text {. }
$$

Let $S=\left[\begin{array}{ll}X & S_{2}\end{array}\right]$ be any nonsingular matrix such that $X$ has $m$ columns and

$$
S^{-1} A S=\left[\begin{array}{cc}
\lambda I_{m} & 0 \\
0 & E
\end{array}\right], \quad E \text { is }(n-m) \times(n-m) .
$$

Then, $\lambda$ is not an eigenvalue of $c E$ or $E$. Partition $\left(S^{-1}\right)^{*}=\left[\begin{array}{ll}Y & Z_{2}\end{array}\right]$, in which $Y$ has $m$ columns. Then $A X=\lambda X, Y^{*} A=\lambda Y^{*}$, and $Y^{*} X=$ $I_{m}$. Moreover, the columns of $X$ may be chosen to be any $m$ linearly independent right $\lambda$-eigenvectors of $A$, and

$$
X Y^{*}=I-(\lambda I-A)(\lambda I-A)^{D}
$$

is a projection that is determined uniquely by $A$ and $\lambda$, regardless of the choice of columns of $X$. Define the vector $y(c)$ by

$$
y(c)^{*}=v^{*} X Y^{*}+(1-c) \lambda v^{*} S_{2}\left(\lambda I_{n-m}-c E\right)^{-1} Z_{2}^{*} .
$$

Then, $y(c)$ satisfies the conditions (5.2); if, in addition, $c \neq 1$, then $y(c)$ is the only vector that satisfies these conditions. If both $A$ and $\lambda$ are real, then $X Y^{*}$ is a real projection. 
(c) Suppose that $\lambda$ is a semisimple eigenvalue of $A$ with multiplicity $m$. Let $K$ be a given compact complex set that does not contain any of the points $\mu_{1}^{-1}, \ldots, \mu_{d}^{-1}$. Let $\tilde{c}$ and $c$ be distinct points in $K$. If $m \geq 2$, let $y(\cdot)$ be defined by (5.6). Then,

$$
\frac{y(\tilde{c})^{*}-y(c)^{*}}{\tilde{c}-c}=\lambda v^{*} S_{2}(\tilde{c} E-\lambda I)^{-1}(E-\lambda I)(c E-\lambda I)^{-1} Z_{2}^{*}
$$

the derivative of $y(c)^{*}$ is

$$
y^{*}=\lambda v^{*} S_{2}(c E-\lambda I)^{-2}(E-\lambda I) Z_{2}^{*}
$$

the derivative of $y(c)^{*}$ at $c=0$ is

$$
y^{\prime *}=\lambda^{-1} v^{*} S_{2}(E-\lambda I) Z_{2}^{*}=\lambda^{-1} v^{*}(A-\lambda I)
$$

and the derivative of $y(c)^{*}$ at $c=1$ is

$$
y^{\prime *}=\lambda v^{*} S_{2}(E-\lambda I)^{-1} Z_{2}^{*}=\lambda v^{*}(A-\lambda I)^{D} .
$$

If $m=1$ and $y(\cdot)$ is defined by (5.1), then the four preceding identities are correct if we replace $E$ with $B, S_{2}$ with $S_{1}$, and $Z_{2}$ with $Z_{1}$. For each given vector norm $\|\cdot\|$ there is a positive constant $M$ (depending on $A, \lambda, v$, and K) such that

$$
\|y(\tilde{c})-y(c)\| \leq M|\tilde{c}-c| \text { for all } \tilde{c}, c \in K
$$

Proof.

(a) The similarity (4.1) shows that the eigenvalues of $B$ are $\lambda_{2}, \ldots, \lambda_{n}$, so our assumption that $\lambda \neq c \lambda_{j}$ for all $j=1, \ldots, n$ ensures that $\lambda$ is not an eigenvalue of $c B$. If $\lambda$ is an eigenvalue of $B$, it must have multiplicity at least two as an eigenvalue of $A$, so if it is a simple eigenvalue of $A$, it is not an eigenvalue of $B$. The vector $y(c)$ defined by (5.1) satisfies the condition $y(c)^{*} x=1$ because $y^{*} x=1$ and $Z_{1}^{*} x=0$. To show that it is a left $\lambda$-eigenvector of $A(c)$, we begin by combining (4.5) and (4.6):

$$
S^{-1}\left(c A+(1-c) \lambda x v^{*}\right) S=\left[\begin{array}{cc}
\lambda & (1-c) \lambda v^{*} S_{1} \\
0 & c B
\end{array}\right]
$$

A calculation verifies that the vector $\eta(c)$ defined by

$$
\eta(c)^{*}=\left[\begin{array}{ll}
1 & (1-c) \lambda v^{*} S_{1}\left(\lambda I_{n-1}-c B\right)^{-1}
\end{array}\right]
$$


is a left $\lambda$-eigenvector of the matrix in (5.12) and that $\eta(c)^{*} e_{1}=1$; if $c \neq 1$, it is the only such vector. Therefore, the vector $y(c)$ defined by

$$
\begin{aligned}
y(c)^{*} & =\eta(c)^{*} S^{-1}=\left[\begin{array}{ll}
1 & (1-c) \lambda v^{*} S_{1}\left(\lambda I_{n-1}-c B\right)^{-1}
\end{array}\right]\left[\begin{array}{c}
y^{*} \\
Z_{1}^{*}
\end{array}\right] \\
& =y^{*}+(1-c) \lambda v^{*} S_{1}\left(\lambda I_{n-1}-c B\right)^{-1} Z_{1}^{*}
\end{aligned}
$$

is a normalized left $\lambda$-eigenvector of $A(c)$, and if $c \neq 1$ it is the only vector that satisfies the conditions (5.2).

(b) Let $D$ denote the block diagonal matrix in (5.4), and let $S$ be any nonsingular matrix such that $S^{-1} A S=D$. Partition $S=\left[X S_{2}\right]$ and $\left(S^{-1}\right)^{*}=$ $\left[\begin{array}{ll}Y & Z_{2}\end{array}\right]$, in which $X$ and $Y$ have $m$ columns. Then,

$$
\left[A X S_{2}\right]=A S=S D=\left[\lambda X S_{2} D\right]
$$

and

$$
\left[\begin{array}{c}
Y^{*} A \\
Z_{2}^{*} A
\end{array}\right]=S^{-1} A=D S^{-1}=\left[\begin{array}{c}
\lambda Y^{*} \\
E Z_{2}^{*}
\end{array}\right],
$$

which tells us that the columns of $X$ are a linearly independent set of right $\lambda$-eigenvectors of $A$ and the columns of $Y$ are a linearly independent set of left $\lambda$-eigenvectors of $A$. The identity $S^{-1} S=I$ tells us that $Y^{*} X=I_{m}$ and hence that $X^{*} Y=\left(Y^{*} X\right)^{*}=I_{m}^{*}=I_{m}$.

Now let $R$ be any given nonsingular $m \times m$ matrix, and let $\hat{S}=\left[\begin{array}{ll}X R & S_{2}\end{array}\right]:=$ $\left[\begin{array}{ll}\hat{X} & S_{2}\end{array}\right]$. Partition $\left(\hat{S}^{-1}\right)^{*}=\left[\begin{array}{ll}\hat{Y} & \hat{Z}_{2}\end{array}\right]$, compute $\left(\hat{S}^{-1}\right)^{*}=\left[\begin{array}{ll}Y\left(R^{-1}\right)^{*} & Z_{2}\end{array}\right]$, and notice that $\hat{Y} \hat{X}^{*}=Y X^{*}$. We draw two conclusions from these observations:

(1) We are free to let the columns of $X$ be any linearly independent set of right $\lambda$-eigenvectors of $A$.

(2) Regardless of the choice of columns of $X$, the product $Y X^{*}$ remains the same. Moreover, $\left(Y X^{*}\right)^{2}=Y\left(X^{*} Y\right) X^{*}=Y I_{m} X^{*}=Y X^{*}$, so $Y X^{*}$ (and hence also $\left.X Y^{*}\right)$ is a projection.

This second conclusion also follows from a useful representation for $X Y^{*}$. We have

$$
\lambda I-A=S\left[\begin{array}{cc}
0 & 0 \\
0 & \lambda I-E
\end{array}\right] S^{-1}
$$

and

$$
(\lambda I-A)^{D}=S\left[\begin{array}{cc}
0 & 0 \\
0 & (\lambda I-E)^{-1}
\end{array}\right] S^{-1},
$$


hence

$$
\begin{aligned}
I-(\lambda I-A)(\lambda I-A)^{D} & =I-S\left[\begin{array}{cc}
0 & 0 \\
0 & I_{n-m}
\end{array}\right] S^{-1} \\
& =\left[X S_{2}\right]\left[\begin{array}{cc}
I_{m} & 0 \\
0 & 0
\end{array}\right]\left[\begin{array}{c}
Y^{*} \\
Z_{2}^{*}
\end{array}\right] \\
& =X Y^{*} .
\end{aligned}
$$

Let the first column of $X$ be the given $\lambda$-eigenvector $x$ that satisfies $v^{*} x=1$, and write $X=\left[\begin{array}{ll}x & \tilde{X}\end{array}\right]$. Then, $x$ is the first column of $S$, so $S^{-1} x=e_{1}$ and

$$
v^{*} S=\left[\begin{array}{lll}
v^{*} X & v^{*} S_{2}
\end{array}\right]=\left[\begin{array}{lll}
v^{*} x & v^{*} \tilde{X} & v^{*} S_{2}
\end{array}\right]=\left[\begin{array}{lll}
1 & v^{*} \tilde{X} & v^{*} S_{2}
\end{array}\right] .
$$

Thus,

$$
\begin{aligned}
S^{-1}\left(x v^{*}\right) S=\left(S^{-1} x\right)\left(v^{*} S\right) & =\left[\begin{array}{l}
1 \\
0 \\
0
\end{array}\right]\left[\begin{array}{ccc}
1 & v^{*} \tilde{X} & v^{*} S_{2}
\end{array}\right] \\
& =\left[\begin{array}{ccc}
1 & v^{*} \tilde{X} & v^{*} S_{2} \\
0 & 0 & 0 \\
0 & 0 & 0
\end{array}\right],
\end{aligned}
$$

and so

$$
S^{-1}\left(c A+(1-c) \lambda x v^{*}\right) S=\left[\begin{array}{ccc}
\lambda & (1-c) \lambda v^{*} \tilde{X} & (1-c) \lambda v^{*} S_{2} \\
0 & c \lambda I_{m-1} & 0 \\
0 & 0 & c E
\end{array}\right] .
$$

The assumption (5.3) (which is satisfied for $c=1$ ) ensures that $\lambda$ is not an eigenvalue of $c E$, and a calculation verifies that $\eta(c)$ defined by

$$
\begin{aligned}
\eta(c)^{*} & =\left[\begin{array}{lll}
1 & v^{*} \tilde{X} & (1-c) \lambda v^{*} S_{2}(\lambda I-c E)^{-1}
\end{array}\right] \\
& =\left[\begin{array}{ll}
v^{*} X(1-c) \lambda v^{*} S_{2}(\lambda I-c E)^{-1}
\end{array}\right]
\end{aligned}
$$

is a left $\lambda$-eigenvector of the matrix in (5.14) and that $\eta(c)^{*} e_{1}=1$; if $c \neq 1$ it is the unique such vector. Therefore, $y(c)$ defined by

$$
\begin{aligned}
y(c)^{*} & =\eta(c)^{*} S^{-1}=\left[\begin{array}{ll}
v^{*} X & (1-c) \lambda v^{*} S_{2}(\lambda I-c E)^{-1}
\end{array}\right]\left[\begin{array}{c}
Y^{*} \\
Z_{2}^{*}
\end{array}\right] \\
& =v^{*} X Y^{*}+(1-c) \lambda v^{*} S_{2}(\lambda I-c E)^{-1} Z_{2}^{*}
\end{aligned}
$$

satisfies the conditions (5.2); if $c \neq 1$ it is the only vector that satisfies these conditions.

If $A$ and $\lambda$ are real, the matrix $S=\left[X S_{2}\right]$ that gives the reduced form (5.4) may be taken to be real (one may reduce to the real Jordan form [Horn and Johnson 85, Theorem 3.4.5]). Then, $\left(S^{-1}\right)^{*}=\left[\begin{array}{ll}Y & Z_{2}\end{array}\right]$ is real, so the uniquely determined product $X Y^{*}$ must always be real, regardless of the choice of $X$. 
(c) Using the identity $\alpha R^{-1}-\beta T^{-1}=R^{-1}(\alpha T-\beta R) T^{-1}$, we compute

$$
\begin{aligned}
y(\tilde{c})^{*}-y(c)^{*} & =\lambda v^{*} S_{2}\left((1-\tilde{c})(\lambda I-\tilde{c} E)^{-1}-(1-c)(\lambda I-c E)^{-1}\right) Z_{2}^{*} \\
& =(\tilde{c}-c) \lambda v^{*} S_{2}(\tilde{c} E-\lambda I)^{-1}(E-\lambda I)(c E-\lambda I)^{-1} Z_{2}^{*} .
\end{aligned}
$$

This identify verifies (5.7). One obtains (5.8) by letting $\tilde{c} \rightarrow c ;(5.9)$ and (5.10) follow by setting $c=1$ and $c=0$, respectively. The bound (5.11) follows from taking the norm of both sides of (5.7) and observing that the right-hand side is a continuous function on a compact set, so it is bounded.

The vector function $y(c)$ defined by (5.6) is a complex analytic function at all but finitely many points in the complex plane, provided that $\lambda$ is a nonzero semisimple eigenvalue of $A$. The points $c=0$ and $c=1$ are of special interest.

- The condition (5.3) is satisfied for all $c$ such that $|c|<\min \left\{\left|\lambda \mu_{j}^{-1}\right|: j=\right.$ $1, \ldots, d\}$. Thus, $y(c)$ is analytic in an open neighborhood of $c=0$ and can be represented there by a Maclaurin series obtained from (5.6) by expanding $\left(\lambda I_{n-m}-c E\right)^{-1}$ as a power series in $c$ :

$$
\begin{aligned}
y(c)^{*}=v^{*} & \left(I+\lambda^{-1}\left(S_{2}(E-\lambda I) Z_{2}^{*}\right) c\right. \\
& \left.+\sum_{k=2}^{\infty} \lambda^{-k}\left(S_{2}(E-\lambda I) E^{k-1} Z_{2}^{*}\right) c^{k}\right) \\
= & v^{*}\left(I+\lambda^{-1}(A-\lambda I) c+\sum_{k=2}^{\infty} \lambda^{-k}\left((A-\lambda I) A^{k-1}\right) c^{k}\right) .
\end{aligned}
$$

This representation reveals all of the derivatives of $y(c)$ at $c=0$.

- The condition (5.3) is also satisfied for all $c$ such that $|c-1|<\min \left\{\mid \lambda \mu_{j}^{-1}-\right.$ $1 \mid: j=1, \ldots, d\}$. Thus, $y(c)$ is analytic in an open neighborhood of $c=1$. If we let $\gamma=c-1$, use (5.6), and expand

$$
(\lambda I-c E)^{-1}=(\lambda I-E)^{-1}\left(I-\gamma E(\lambda I-E)^{-1}\right)^{-1}
$$

as a power series in $\gamma$, we obtain

$$
\begin{aligned}
y(\gamma+1)^{*}= & v^{*}\left(X Y^{*}-\lambda S_{2}(\lambda I-E)^{-1} Z_{2}^{*} \gamma\right. \\
& \left.-\lambda \sum_{k=2}^{\infty}\left(S_{2}(\lambda I-E)^{-k} E^{k-1} Z_{2}^{*}\right) \gamma^{k}\right) .
\end{aligned}
$$


This series reveals all the derivatives of $y(c)$ at $c=1$. We can use the Drazin inverse to write this series as

$$
\begin{aligned}
y(\gamma+1)^{*}= & v^{*}\left(X Y^{*}-\lambda(\lambda I-A)^{D} \gamma\right. \\
& \left.-\lambda \sum_{k=2}^{\infty}\left(\left((\lambda I-A)^{D}\right)^{k} A^{k-1}\right) \gamma^{k}\right) .
\end{aligned}
$$

In particular, the first derivative at $c=1$ is

$$
y^{\prime *}=\lambda v^{*} S_{2}(E-\lambda I)^{-1} Z_{2}^{*}=\lambda v^{*}(A-\lambda I)^{D} .
$$

\section{The Behavior of $y(c)$ as $c \rightarrow 1$}

We are interested in the behavior of the left eigenvector $y(c)$ defined by (5.1) as $c \rightarrow 1$ in the complex plane, and to understand it better, we consider two examples.

- Example 1. Consider

$$
A=\left[\begin{array}{lll}
1 & 0 & 0 \\
0 & 1 & 0 \\
0 & 0 & 2
\end{array}\right], \lambda=1, x=y=e_{1}, v^{*}=\left[\begin{array}{lll}
1 & \alpha & \beta
\end{array}\right] .
$$

The hypotheses of Theorem 5.1 are satisfied for all complex $c \notin\{1,1 / 2\}$, and the vector $y(c)$ defined by

$$
y(c)^{*}=\left[\begin{array}{lll}
1 & \alpha & \frac{(c-1) \beta}{2 c-1}
\end{array}\right]
$$

is the normalized left eigenvector of

$$
c A+(1-c) \lambda x v^{*}=\left[\begin{array}{ccc}
1 & (1-c) \alpha & (1-c) \beta \\
0 & c & 0 \\
0 & 0 & 2 c
\end{array}\right]
$$

associated with the eigenvalue $\lambda=1$. Moreover,

$$
\lim _{c \rightarrow 1} y(c)^{*}=\left[\begin{array}{lll}
1 & \alpha & 0
\end{array}\right] .
$$

Although $\lambda=1$ is not a simple eigenvalue of $A$, it is semisimple.

- Example 2. Consider

$$
A=\left[\begin{array}{lll}
1 & 0 & 0 \\
0 & 1 & 1 \\
0 & 0 & 1
\end{array}\right], \lambda=1, x=y=e_{1}, v^{*}=\left[\begin{array}{lll}
1 & \alpha & \beta
\end{array}\right] .
$$


The hypotheses of Theorem 5.1 are satisfied for all complex $c \neq 1$, and the vector $y(c)$ defined by

$$
y(c)^{*}=\left[\begin{array}{lll}
1 & \alpha & \beta+\frac{c \alpha}{1-c}
\end{array}\right]
$$

is the normalized left eigenvector of

$$
c A+(1-c) \lambda x v^{*}=\left[\begin{array}{ccc}
1 & (1-c) \alpha & (1-c) \beta \\
0 & c & c \\
0 & 0 & c
\end{array}\right]
$$

associated with the eigenvalue $\lambda=1$. However, $\lim _{c \rightarrow 1} y(c)^{*}$ does not exist unless $\alpha=0$. In this case, $\lambda=1$ is not semisimple.

These two examples are not exceptional: when $\lambda \neq 0$, semisimplicity of $\lambda$ is the essential hypothesis required to ensure that $\lim _{c \rightarrow 1} y(c)$ exists for all choices of $v$. The following theorem verifies this assertion and gives an explicit formula for the limit.

Theorem 6.I. Let $A$ be an $n \times n$ complex matrix with eigenvalues $\lambda, \lambda_{2}, \ldots, \lambda_{n}$. Suppose that $\lambda$ is a nonzero semisimple eigenvalue of $A$ with multiplicity $m \geq 1$; let $x$ and $v$ be given nonzero complex vectors such that $A x=\lambda x$ and $v^{*} x=1$; and let $A(c)=c A+(1-c) \lambda x v^{*}$. If $m=1$, let $y$ be the unique vector such that $y^{*} A=\lambda y$ and $y^{*} x=1$. If $m>1$, let $X Y^{*}=I-(\lambda I-A)(\lambda I-A)^{D}$ be the projection defined in Theorem 5.1(b). Then,

(a) For some $\varepsilon>0$ and all complex $c$ such that $0<|c-1|<\varepsilon$, as well as for all complex $c$ such that $\lambda \neq c \lambda_{j}$ for all $j=2, \ldots, n$, the vector $y(c)$ defined by (5.1) when $\lambda$ is simple, or by (5.6) when it is not, is the unique vector that satisfies $y(c)^{*} A(c)=\lambda y(c)^{*}$ and $y(c)^{*} x=1$.

(b) If $\lambda$ is a simple eigenvalue of $A$, then $\lim _{c \rightarrow 1} y(c)=y x^{*} v=y$.

(c) If $m>1$, then

$$
\lim _{c \rightarrow 1} y(c)=Y X^{*} v=\left(X Y^{*}\right)^{*} v
$$

Proof.

(a) If $\lambda$ and 0 are the only eigenvalues of $A$, then any positive value of $\epsilon$ will do. If the nonzero eigenvalues of $A$ that are different from $\lambda$ are $\mu_{1}, \ldots, \mu_{d}$, let

$$
\epsilon=\min \left\{\left|1-\lambda \mu_{1}^{-1}\right|, \ldots,\left|1-\lambda \mu_{d}^{-1}\right|\right\} .
$$


Then the hypothesis (4.7) is satisfied and the assertion follows from Theorem 5.1. Since $y(c)$ is defined in a punctured open complex neighborhood of the point $c=1$, it is reasonable to ask about the limit of $y(c)$ (as a function of the complex variable $c$ ) as $c \rightarrow 1$.

(b) The assertion follows from (5.1) since $\lambda$ is not an eigenvalue of $B$ :

$$
\begin{aligned}
\lim _{c \rightarrow 1} y^{*}(c) & =y^{*}+\lim _{c \rightarrow 1}\left((1-c) \lambda v^{*} S_{1}(\lambda I-c B)^{-1} Z_{1}^{*}\right) \\
& =y^{*}+\lim _{c \rightarrow 1}(1-c) \cdot \lambda v^{*} S_{1} \cdot \lim _{c \rightarrow 1}(\lambda I-c B)^{-1} Z_{1}^{*} \\
& =y^{*}+\left(0 \cdot \lambda v^{*} S_{1}(\lambda I-B)^{-1} Z_{1}^{*}\right)=y^{*}=v^{*} x y^{*} .
\end{aligned}
$$

(c) This assertion follows in the same way from (5.6):

$$
\begin{aligned}
\lim _{c \rightarrow 1} y(c)^{*} & =v^{*} X Y^{*}+\lim _{c \rightarrow 1}\left((1-c) \lambda v^{*} S_{2}\left(\lambda I_{n-m}-c E\right)^{-1} Z_{2}^{*}\right) \\
& =v^{*} X Y^{*}+\lim _{c \rightarrow 1}(1-c) \cdot \lambda v^{*} S_{2} \cdot \lim _{c \rightarrow 1}\left(\lambda I_{n-m}-c E\right)^{-1} Z_{2}^{*} \\
& =v^{*} X Y^{*}+0 \cdot \lambda v^{*} S_{2} \cdot\left(\lambda I_{n-m}-E\right)^{-1} Z_{2}^{*}=v^{*} X Y^{*} .
\end{aligned}
$$

\section{The Projection $\boldsymbol{X} \boldsymbol{Y}^{*}$ is an Important Special Case}

In (5.5) we have two different representations for the projection $X Y^{*}$. A third representation is available under special conditions that are satisfied by the Google matrix.

Theorem 7.I. Let $A$ be an $n \times n$ complex matrix. Suppose that the spectral radius of $A$ is positive and that every eigenvalue $\nu$ of $A$ such that $|\nu|=\rho(A)$ is semisimple. Let $\lambda$ be an eigenvalue of $A$ with multiplicity $m$ and assume that $|\lambda|=\rho(A)$. If $m=1$, let $x$ and $y$ be right and left, respectively, $\lambda$-eigenvectors of $A$, normalized so that $y^{*} x=1$; let $X Y^{*}:=x y^{*}$. If $m>1$, let $X Y^{*}$ be the projection defined in Theorem 5.1(b). Then,

$$
X Y^{*}=\lim _{N \rightarrow \infty} \frac{1}{N} \sum_{k=0}^{N-1}\left(\lambda^{-1} A\right)^{k}
$$

Proof. Write $\lambda=e^{i \theta_{0}} \rho(A)$ and suppose that $\lambda$ has multiplicity $m$. Let $\nu_{1}, \ldots, \nu_{p}$ be the distinct eigenvalues of $A$ that are different from $\lambda$ and have modulus $\rho(A)$; write $\nu_{j}=e^{i\left(\theta_{j}+\theta_{0}\right)} \rho(A), j=1, \ldots, p$, and suppose that each $\nu_{j}$ has multiplicity $m_{j}$. Write the Jordan canonical form of $A$ as

$$
A=S\left(\lambda I_{m} \oplus \nu_{1} I_{m_{1}} \oplus \cdots \oplus \nu_{p} I_{m_{p}} \oplus J\right) S^{-1},
$$


in which $J$ is a direct sum of Jordan blocks whose eigenvalues have modulus strictly less than $\rho(A), S=\left[\begin{array}{ll}X & S_{2}\end{array}\right],\left(S^{-1}\right)^{*}=\left[\begin{array}{ll}Y & Z_{2}\end{array}\right]$, and each of $X$ and $Y$ has $m$ columns. Notice that $\left(\lambda^{-1} J\right)^{N} \rightarrow 0$ as $N \rightarrow \infty$ [Horn and Johnson 85, Section 3.2.5]. The identity

$$
\sum_{k=0}^{N-1} B^{k}=\left(I-B^{N}\right)(I-B)^{-1}
$$

is valid whenever $I-B$ is nonsingular. If we employ this identity with $B=\lambda^{-1} J$ or $B=\lambda^{-1} \nu_{j} I_{m_{j}}=e^{i \theta_{j}} I_{m_{j}}$, we find in either case that $\sum_{k=0}^{N-1} B^{k}$ is bounded as $N \rightarrow \infty$, which ensures that $N^{-1} \sum_{k=0}^{N-1} B^{k} \rightarrow 0$ as $N \rightarrow \infty$. Thus,

$$
\lim _{N \rightarrow \infty} \frac{1}{N} \sum_{k=0}^{N-1}\left(\lambda^{-1} A\right)^{k}=S\left(I_{m} \oplus 0_{m_{1}} \oplus \cdots \oplus 0_{m_{p}} \oplus 0\right) S^{-1}=X Y^{*} .
$$

When the theorem's hypotheses about $A$ 's eigenvalues of maximum modulus are satisfied, the representation (7.1) reveals two important facts about the projection $X Y^{*}$ : (a) if $\lambda$ and $A$ are real, then $X Y^{*}$ is real (we have a more general result in Theorem 5.1(b)); and (b) if $\lambda>0$ and the entries of $A$ are nonnegative, then the entries of $X Y^{*}$ are nonnegative.

\section{A Special Case: The General Parametric Google Matrix}

We begin with a summary of the properties of a row-stochastic matrix that are relevant to our analysis of the general parametric Google matrix.

Lemma 8.I. Let $A$ be a row-stochastic matrix. Then,

(a) $\lambda=1$ is an eigenvalue of $A$ associated with the right eigenvector $x=e$;

(b) every entry of $A$ is in the real interval $[0,1]$;

(c) for each $k=1,2, \ldots, A^{k}$ is row stochastic, so its entries remain bounded as $k \rightarrow \infty$;

(d) every eigenvalue of $A$ has modulus at most 1;

(e) every eigenvalue of $A$ that has modulus 1 is semisimple;

(f) if the eigenvalue 1 has multiplicity $m$, then the Jordan canonical form of $A$ is

$$
I_{m} \oplus J_{n_{1}}\left(\nu_{1}\right) \oplus \cdots \oplus J_{n_{k}}\left(\nu_{k}\right),
$$

in which each $\nu_{j} \neq 1$, each $\left|\nu_{j}\right| \leq 1$, and $n_{j}=1$ if $\left|\nu_{j}\right|=1$; 
(g) if 1 is a simple eigenvalue of $A$, then there is a unique vector $y$ with nonnegative entries such that $y^{T} A=y^{T}$ and $y^{T} e=1$.

Proof.

(a) Every row sum of $A$ is one: $A e=e$.

(b) The sum of the (nonnegative) entries in each row of $A$ is 1 , so no entry can be greater than 1 .

(c) Compute $A^{2} e=A(A e)=A e=e$ and proceed by induction to show that $A^{k}$ is row stochastic for all $k=1,2, \ldots$ Then (a) ensures that the entries of each $A^{k}$ are in $[0,1]$.

(d)-(f) Suppose that

$$
J=J_{n_{1}}\left(\lambda_{1}\right) \oplus \cdots \oplus J_{n_{r}}\left(\lambda_{r}\right)
$$

is the Jordan canonical form of $A$ and that $A=S J S^{-1}$. It follows from (c) that the entries of $J^{k}=S^{-1} A^{k} S$ remain bounded as $k \rightarrow \infty$. Each main diagonal entry of $J_{n_{i}}\left(\lambda_{i}\right)^{k}$ is $\lambda_{i}^{k}$, which remains bounded as $k \rightarrow \infty$ only if $\left|\lambda_{i}\right| \leq 1$. If $n_{i}>1$, one checks that each of the $n_{i}-1$ super-diagonal entries of $J_{n_{i}}\left(\lambda_{i}\right)^{k}$ is $k \lambda_{i}^{k-1}$, which is unbounded as $k \rightarrow \infty$ if $\left|\lambda_{i}\right|=1$. Thus, every Jordan block associated with any eigenvalue with modulus 1 must be $1 \times 1$, that is, every eigenvalue with modulus 1 is semisimple. For a different proof, see [Meyer 00, p. 696]

(g) The Perron-Frobenius Theorem [Horn and Johnson 85, Theorem 8.3.1] ensures that there is a unique nonnegative (and by definition nonzero) left eigenvector $y$ associated with the eigenvalue 1 that is normalized so that $y^{T} e=1$.

Since the basic Google matrix $G$ has all the properties stated in the preceding lemma, and since these properties are special cases of the key hypotheses in our analyses in the preceding sections, specialization of our general results permits us to identify several pleasant and useful properties of the general parametric Google matrix $G(c)=c G+(1-c) x v^{*}$ with complex $c$ and $v$.

Theorem 8.2. Let $G$ be an $n \times n$ row-stochastic matrix, and let its eigenvalue $\lambda=1$ (necessarily semisimple) have multiplicity $m \geq 1$. If $m=1$, let $y$ be the unique vector with nonnegative entries such that $y^{T} G=y^{T}$ and $y^{T} e=1$. If $m>1$, let the $m$ columns of $X$ be any linearly independent set of right eigenvectors of $G$ associated with the eigenvalue $\lambda=1$, and let $Y$ be the matrix defined in Theorem $5.1(b)$; its $m$ columns are an independent set of left eigenvectors of $G$ associated with the eigenvalue $\lambda=1$. Let $v$ be a given complex vector such that $v^{*} e=1$, 
let $c$ be a complex number, and let $G(c)=c G+(1-c) e v^{*}$. Let $1, \lambda_{2}, \ldots, \lambda_{n}$ be the eigenvalues of $G$, let $\mu_{1}, \ldots, \mu_{d}$ be the nonzero eigenvalues of $G$ that are different from 1 , let

$$
\epsilon=\min \left\{\left|1-\mu_{1}^{-1}\right|, \ldots,\left|1-\mu_{d}^{-1}\right|\right\}
$$

and let

$$
I_{m} \oplus J_{n_{1}}\left(\nu_{1}\right) \oplus \cdots \oplus J_{n_{k}}\left(\nu_{k}\right) \text {, each } \nu_{j} \neq 1
$$

be the Jordan canonical form of $G$. Then, each of the following statements is true:

(a) The eigenvalues of $G(c)$ are $1, c \lambda_{2}, \ldots, c \lambda_{n}$ and $\left|c \lambda_{j}\right| \leq|c|$ for each $j=$ $2, \ldots, n$.

(b) In the Jordan canonical form (8.1), $n_{j}=1$ for each $j$ such that $\left|\nu_{j}\right|=1$.

(c) If $0<|c|<1$ (or, more generally, if $c \neq 0$ and $1 \neq c \nu_{j}$ for each $j=$ $1, \ldots, d)$, then the Jordan canonical form of $G(c)$ is

$$
[1] \oplus c I_{m-1} \oplus J_{n_{1}}\left(c \nu_{1}\right) \oplus \cdots \oplus J_{n_{k}}\left(c \nu_{k}\right)
$$

if $m>1$; it is

$$
[1] \oplus J_{n_{1}}\left(c \nu_{1}\right) \oplus \cdots \oplus J_{n_{k}}\left(c \nu_{k}\right)
$$

if $m=1$.

(d) Suppose either that $|c|<1$ or that $0<|1-c|<\epsilon$. Then, 1 is a simple eigenvalue of $G(c)$.

(e) Suppose either that $|c|<1$ or that $0<|1-c|<\epsilon$. If $m>1$, the unique left 1-eigenvector $y(c)$ of $G(c)$ such that $y(c)^{*} e=1$ is defined by

$$
y(c)^{*}=v^{*} X Y^{*}+(1-c) v^{*} S_{2}\left(I_{n-m}-c E\right)^{-1} Z_{2}^{*},
$$

and

$$
\lim _{c \rightarrow 1} y(c)=Y X^{*} v .
$$

The matrices $S_{2}, E$, and $Z_{2}$ are defined in Theorem 5.1(b); 1 is not an eigenvalue of $E$. The matrix

$$
X Y^{*}=I-(I-G)(I-G)^{D}=\lim _{N \rightarrow \infty} \frac{1}{N} \sum_{k=0}^{N-1} G^{k}
$$

is a real projection with nonnegative entries. 
(f) Suppose either that $|c|<1$ or that $0<|1-c|<\epsilon$. If $m=1$, the unique left 1-eigenvector $y(c)$ of $G(c)$ such that $y(c)^{*} e=1$ is defined by

$$
y(c)^{*}=y^{*}+(1-c) v^{*} S_{1}\left(I_{n-1}-c B\right)^{-1} Z_{1}^{*},
$$

and

$$
\lim _{c \rightarrow 1} y(c)=y \text {. }
$$

The matrices $S_{1}, Z_{1}$, and $B$ are defined in Theorem 5.1(a); 1 is not an eigenvalue of $B$.

(g) The vector function $y(c)$ defined by (8.2) if $m>1$ and by (8.5) if $m=1$ is analytic in the unit disk $\{c:|c|<1\}$ and is represented there by the Maclaurin series

$$
y(c)^{*}=v^{*}\left(I+(G-I) c+\sum_{k=2}^{\infty}\left((G-I) G^{k-1}\right) c^{k}\right) .
$$

(h) Let $\gamma=c-1$. The vector function $y(c)$ defined by (8.2) if $m>1$, and by (8.5) if $m=1$, is analytic in the disk $\{c:|1-c|<\epsilon\}$ and is represented there by the power series

$$
y(c)^{*}=y(\gamma+1)^{*}=v^{*}\left(X Y^{*}-(I-G)^{D} \gamma-\sum_{k=2}^{\infty}\left(\left((I-G)^{D}\right)^{k} G^{k-1}\right) \gamma^{k}\right) .
$$

In particular, the first derivative at $c=1$ is

$$
y^{\prime *}=v^{*}(G-I)^{D}
$$

(i) Let $K$ be a given compact complex set that does not contain any of the points $\mu_{1}^{-1}, \ldots, \mu_{d}^{-1}$. Define $y(c)$ on $K$ by (8.2) if $m>1$ and by (8.5) if $m=1$. Then, $\|y(c)\|_{1} \geq 1$ for all $c \in K$, and there is a positive constant $M$ such that

$$
\frac{\|y(\tilde{c})-y(c)\|_{1}}{\|y(c)\|_{1}} \leq\|y(\tilde{c})-y(c)\|_{1} \leq M|\tilde{c}-c| \text { for all } \tilde{c}, c \in K
$$

The representations for $X Y^{*}$ in (8.4) are special cases of (5.5) and (7.1). The assertions in (g) and (h) follow from (5.16), (5.18), and (5.19). The assertion (i) follows from Theorem 5.1(c) and the observation that $1=\left|y(c)^{*} e\right| \leq\left\|y(c)^{*}\right\|_{1}$.

We emphasize that the representations (8.2) and (8.5) for the unique normalized left 1-eigenvector of $G(c)$ are valid not only for all real $c \in(0,1)$ but also for all complex $c$ in the open unit disk, as well as for all $c$ in a punctured open 
neighborhood of the point 1 in the complex plane and for all $c$ such that $|c| \geq 1$ and $c \neq \mu_{j}^{-1}$ for all $j=1, \ldots, d$. The limits (8.3) and (8.6) are to be understood as limits of functions of a complex variable; the existence of these limits ensures that they may be computed via any sequence of real or complex values of $c$ that tend to 1 .

The projection matrix $Y X^{*}=\left[\begin{array}{lll}\eta_{1} & \ldots & \eta_{n}\end{array}\right]$ is real and nonnegative. Its columns are a uniquely determined set of nonnegative left 1-eigenvectors of $G$ such that, for any given probability vector $v=\left[v_{i}\right], \lim _{c \rightarrow 1} y(c)=v_{1} \eta_{1}+\cdots+v_{n} \eta_{n}$ is a convex combination of them.

Estimates given in [Kirkland 06] suggest that $y(c)$ might be poorly conditioned near $c=1$; indeed, some of those estimates contain a factor $(1-c)^{-1}$. But our analysis shows that $y(c)$ has a natural definition at $c=1$ that makes it analytic in an open neighborhood of $c=1$.

It has been said that the "PageRank problem is closely related to Markov chains" [Brezinski and Zaglia 06, p. 553]. However, framing the PageRank problem in the general setting of standard matrix-analytic properties of complex matrices can liberate one's imagination and stimulate novel approaches that might not be considered in the context of Markov chains.

\section{Computational Suggestions}

We propose the following algorithm for experimentation and further research.

Choose a positive integer $p$ (let us say, $p=10$ ), and use the power method to compute the left 1-eigenvectors $y_{j}:=y\left(r e^{2 \pi \mathrm{i} j / p}\right), j=0, \ldots, p-1$, at $p$ equally-spaced points on the complex circle of (small) radius $r$ (let us say, $r=0.5$ or 0.25$)$. These computations are extremely fast since the standard power method at the $k$ th iteration converges with a relative reduction error of at least $r^{k}$ [Golub and Van Loan 83, p. 330]. Moreover, one could employ a vector-valued Fast Fourier Transform procedure, whose numerical stability is excellent.

Now use the $p$ computed vectors $y_{0}, \ldots, y_{p-1}$ as a starting point for an extrapolation algorithm to compute (an approximation to) $y(c)$ at $c=0.85$ (or even $c=0.99)$ [Brezinski and Zaglia 06, Brezinski et al. 05]. The idea is to choose linear combinations of $y_{0}, \ldots, y_{p-1}$ that zero out certain terms in an expansion of the remainder $y(c)-Y X^{*} v$ [Brezinski and Zaglia 91, Chapter 4].

Now extract the real part of $y(c)$, set to zero any entries that are negative, and normalize so that the sum of the entries (the $l_{1}$ norm) is 1 . Finally do iterative refinement to increase precision [Del Corso et al. 05, Ipsen and S. Kirkland 06, Langville and Meyer 05].

Computing the PageRank with $c$ very close to 1 is difficult by straightforward techniques, due to slow convergence (see [Del Corso et al. 05] - and references therein-and [Langville and Meyer 04, Section 6.1] for a discussion of the case 
$c=0.99)$. It will be interesting to see whether an algorithm that exploits complex parameters will work well in practice. The results of numerical experiments for $n$ of moderate size have been promising. (See also [Brezinski and Zaglia 08].)

We summarize the proposed algorithm as follows:

- Step 1: Compute $y\left(c_{j}\right), c_{j}=0.25 * \exp (\mathbf{i} 2 j \pi / p), j=0, \ldots, p-1$ via vector FFT.

- Step 2: Do vector extrapolation at the desired (difficult) $c \approx 1$ (e.g., $c=$ $0.85, c=0.99, c=1)$ to obtain a computed approximation $\tilde{y}(c)$ to $y(c)$.

- Step 3: Project $\tilde{y}(c)$ into the real nonnegative cone and do $l_{1}$ normalization.

- Step 4: Apply iterative refinement by classical procedures. Since $c \approx 1$, it is advisable to use preconditioning and Krylov techniques [Del Corso et al. 05].

\section{Some Comments about Prior Work}

The eigenvalues of the standard real parametric Google matrix $G(c)$ were analyzed by Haveliwala and Kamvar (2003; only the second eigenvalue), Eldén [Eldén 06, Proposition 6.3], and Langville and Meyer [Langville and Meyer 04] (their proof is the same as that of Reams). A different approach via the characteristic polynomial is suggested by Meyer [Meyer 00, Problem 7.1.17, p. 502]. These authors were apparently unaware of the prior work of Brauer [Brauer 52] and Reams [Reams 96].

Relying on sophisticated results about Markov chains, Serra-Capizzano gives an analysis of the Jordan canonical form of the standard real $G(c)$; he also gives a rational representation for $y(c)$ and computes its limit as $c \rightarrow 1$, again in the standard real case only [Serra-Capizzano 05]. The Maclaurin series for $y(c)$ was studied by Boldi, Santini, and Vigna (2005), who identified the partial sums of (8.7) for nonnegative real $v$ and $0<c<1$ as the iterates obtained in solving $y^{T} G(c)=y^{T}$ with the power method starting at $v$.

Boldi et al. obtained the special case (8.3) of our general result (6.1) [Boldi et al. 06]. The matrix $Y X^{*}$ in (8.3) has been called the ergodic projection [Lasserre 94].

Acknowledgments. We acknowledge with thanks many fruitful discussions with Ilse Ipsen, Sebastiano Vigna, and Hugo Zaragoza. The research discussed in this paper was stimulated by a remark that Gene Golub, to whom this paper is dedicated, made while lecturing at the University of Utah on November 11, 2005. It is a special pleasure to congratulate him on the occasion of his 75 th birthday and to thank him for many years of mentoring and friendship. 


\section{References}

[Boldi et al. 06] P. Boldi, V. Lonati, M. Santini, and S. Vigna. "Graph Fibrations, Graph Isomorphism, and PageRank." Theor. Inform. Appl. 40 (2006), 227-253.

[Brauer 52] A. Brauer. "Limits for the Characteristic Roots of a Matrix. IV: Applications to Stochastic Matrices." Duke Math. J. 19 (1952), 75-91.

[Brezinski and Zaglia 91] C. Brezinski and M. Redivo Zaglia. Extrapolation Methods: Theory and Practice. Amsterdam: North-Holland, 1991.

[Brezinski and Zaglia 06] C. Brezinski and M. Redivo Zaglia. "The PageRank Vector: Properties, Computation, Approximation, and Acceleration." SIAM J. Matrix Anal. Appl. 28 (2006), 551-575.

[Brezinski and Zaglia 08] C. Brezinski and M. Redivo Zaglia. "Rational Extrapolation for the PageRank Vector." Math. Comput., posted on Februrary7, 2008 PII S 0025-5718(08)02086-3 (to appear in print).

[Brezinski et al. 05] C. Brezinski, M. Redivo Zaglia, and S. Serra-Capizzano. "Extrapolation Methods for PageRank Computations." Comptes Rendus Acad. Sci. Paris - Ser. I 340 (2005), 393-397.

[Campbell and Meyer 79] S. Campbell and C. Meyer. Generalized Inverses of Linear Transformations. New York: Dover Publications, 1979.

[Del Corso et al. 05] G. Del Corso, A. Gullí, and F. Romani. "Fast PageRank Computation via a Sparse Linear System." Internet Math. 2:3 (2005), 251-273.

[Eldén 06] L. Eldén. "Numerical Linear Algebra in Data Mining." Acta Numerica 15 (2006), 327-384.

[Golub and Van Loan 83] G. Golub and C. Van Loan. Matrix Computations. Baltimore: Johns Hopkins University Press, 1983.

[Horn and Johnson 85] R. Horn and C. Johnson. Matrix Analysis. New York: Cambridge University Press, 1985.

[Ipsen and S. Kirkland 06] I. Ipsen and S. Kirkland. "Convergence Analysis of a PageRanking Updating Algorithm by Langville and Meyer." SIAM J. Matrix Anal. Appl. 27:4 (2006), 952-967.

[Kirkland 06] S. Kirkland. "Conditioning of the Entries in the Stationary Vector of a Google-Type Matrix." Linear Algebra Appl. 418 (2006), 665-681.

[Langville and Meyer 04] A. Langville and C. Meyer. "Deeper Inside PageRank." Internet Math. 1:3 (2004), 335-380.

[Langville and Meyer 05] A. Langville and C. Meyer. "A Survey of Eigenvector Methods for WEB Information Retrieval. SIAM Review 47:1 (2005), 135-161.

[Lasserre 94] J. Lasserre. "A Formula for Singular Perturbations of Markov Chains." J. Appl. Prob. 31 (1994), 829-833.

[Meyer 00] C. Meyer. Matrix Analysis and Applied Linear Algebra. Philadelphia: SIAM, 2000.

[Reams 96] R. Reams. "An Inequality for Nonnegative Matrices and the Inverse Eigenvalue Problem." Linear Multilinear Algebra 41 (1996), 367-375. 
[Serra-Capizzano 05] S. Serra-Capizzano. "Jordan Canonical Form of the Google Matrix: A Potential Contribution to the PageRank Computation." SIAM J. Matrix Anal. Appl. 27 (2005), 305-312.

Roger A. Horn, Mathematics Department, University of Utah, 155 South 1400 East, Salt Lake City, UT 84112, USA (rhorn@math.utah.edu)

Stefano Serra-Capizzano, Dipartimento di Fisica e Matematica, Università dell'Insubria - Sede di Como, Via Valleggio 11, 22100 Como, Italy (stefano.serrac@uninsubria.it)

Received March 1, 2007; accepted July 5, 2007. 
